\title{
Introducing a Novel Hybrid Artificial Intelligence Algorithm to Optimize Network of Industrial Applications in Modern Manufacturing
}

\author{
Aydin Azizi \\ Department of Engineering, German University of Technology, Muscat, Oman \\ Correspondence should be addressed to Aydin Azizi; aydin.azizi@gutech.edu.om
}

Received 25 July 2016; Accepted 4 May 2017; Published 14 June 2017

Academic Editor: Alicia Cordero

Copyright (c) 2017 Aydin Azizi. This is an open access article distributed under the Creative Commons Attribution License, which permits unrestricted use, distribution, and reproduction in any medium, provided the original work is properly cited.

Recent advances in modern manufacturing industries have created a great need to track and identify objects and parts by obtaining real-time information. One of the main technologies which has been utilized for this need is the Radio Frequency Identification (RFID) system. As a result of adopting this technology to the manufacturing industry environment, RFID Network Planning (RNP) has become a challenge. Mainly RNP deals with calculating the number and position of antennas which should be deployed in the RFID network to achieve full coverage of the tags that need to be read. The ultimate goal of this paper is to present and evaluate a way of modelling and optimizing nonlinear RNP problems utilizing artificial intelligence (AI) techniques. This effort has led the author to propose a novel AI algorithm, which has been named "hybrid AI optimization technique," to perform optimization of RNP as a hard learning problem. The proposed algorithm is composed of two different optimization algorithms: Redundant Antenna Elimination (RAE) and Ring Probabilistic Logic Neural Networks (RPLNN). The proposed hybrid paradigm has been explored using a flexible manufacturing system (FMS), and results have been compared with Genetic Algorithm (GA) that demonstrates the feasibility of the proposed architecture successfully.

\section{Introduction}

Steady state industry has been changed to dynamic industry by recent industrial revolutions, which has resulted in manufacturers being pushed by the global market to reconsider their conventional manufacturing methods [1]. Modern manufacturing needs new manufacturing operations, and effective factory management has great value in this area [2]. Recent advances in technology and modern industrial engineering systems from production to transportation have created a great need to track and identify the materials, products, and even live subjects [1]. Radio Frequency Identification (RFID) technology is a reliable and efficient solution to this tracking and identifying problem. RFID technology is known as an automatic identification technology as it uses wireless radio frequency waves which are produced by an electromagnetic field to transfer data to track and identify objects. This technology can be implemented in different fields such as tracking and identifying patients in hospitals [2], warehouse item tracking [3], tracking pallets and cases in shipments [4], monitoring production lines [5], and supply chain management [6].

In many applications, the deployment of RFID systems has generated an RFID Network Planning (RNP) problem [7], which needs to be resolved in order to efficiently operate a large-scale network. However, RNP is a very challenging problem, and the solution has to meet many requirements of the RFID system [8, 9]. In general, RNP aims to optimize a set of objectives (coverage, load balance, economic efficiency and interference between antennas, etc.) simultaneously; this is achieved by adjusting the control variables (the coordinates of the readers, the number of antenna, etc.) of the system. As a result, in a large-scale deployment environment, the RNP problem is a multidimensional nonlinear optimization problem that has a vast number of variables and uncertain parameters.

Tracking and identifying objects in these applications require the deployment of several RFID antennas in the RNP, 
and the numbers of these antennas are calculated through the use of a mathematical model [10-12]. In the past, one of the typical ways to address the RNP problem was to use a trial and error approach, which was an inaccurate and inefficient solution for such an important issue [7, 13, 14]. In addition, this approach could only be used on smallscale RFID Network Planning problems [10]. Nowadays, with the developments in computer technology, and software engineering, the conventional trial and error approach has been replaced with modern computational techniques that provide important criteria such as the coverage of objects, collision of antennas, and number of antennas [15]. Computational evolutionary techniques such as Artificial Neural Networks [16], Fuzzy Logic [17], Genetic Algorithms (GA) $[10,11,18]$, particle swarm optimization (PSO) $[13,19,20]$, differential evolution (DE) [9], and hierarchical artificial bee colony algorithm [8] are points of interest for many scientists working with the RNP problem. In this respect, Han and Jie [21] proposed a novel optimization algorithm, namely, the multicommunity GA-PSO, for solving the problem of complicated RNP. Chen et al. [7] developed an optimization model for planning the positions of the readers in the RNP based on PS2O. Nawawi et al. [22] proposed a solution based on the PSO correlation between RNP parameters. Lu and Yu [23] proposed an approach based on $k$-coverage for RFID Network Planning; a $k$-coverage model is formulated from a multidimensional optimization problem and plant growth simulation algorithm. This is used to optimize the RFID networks by determining the optimal adjustable parameters. Furthermore, Gong et al. [13] used the PSO algorithm with redundant reader elimination for optimizing the RNP.

The aim of this paper is to present and evaluate a novel way of optimizing nonlinear RNP problems by utilizing artificial intelligence techniques. Artificial Neural Network (ANN) models are used to bind together the computational artificial intelligence algorithm with knowledge representation of an efficient artificial intelligence paradigm, in order to optimize nonlinear RNP problems. To begin with, existing Artificial Neural Networks models are introduced, which defines the structure that is required in order to optimize functions. Different artificial intelligence algorithms that can satisfy the requirements for optimizing the defined RFID Network Planning problem, which can be represented as mathematical models, are presented and discussed. This effort has led to the proposal of a novel artificial intelligence algorithm, which has been named "hybrid artificial intelligence optimization technique," to perform optimization of RNP as a hard learning problem. The proposed hybrid optimization technique has been made of two different optimization phases. The first phase is optimizing RNP by using the Redundant Antenna Elimination (RAE) algorithm and the second phase, which completes the RNP optimization process, is Ring Probabilistic Logic Neural Networks (RPLNN).

The research developed uses Artificial Neural Network (ANN) models to bind together the computational artificial intelligence algorithm with knowledge representation of an efficient artificial intelligence paradigm, in order to optimize the deployed number of antennas in the RFID network, based on the criteria of defined RNP as a nonlinear engineering problem. Starting from the definition of Radio Frequency Identification systems, which defines the challenges of establishing an efficient RFID network and introduces existing RNP models, what follows is the introduction of existing Artificial Neural Networks models; this defines which structures are required in order to optimize nonlinear RNP functions.

The ultimate goal of this research is to introduce a hybrid artificial intelligence optimization algorithm as a time efficient and reliable optimization technique to solve RFID Network Planning problems and to design a cost effective RFID network by minimizing number of embedded RFID antennas in the network, minimizing collision of antennas and maximizing coverage area of objects.

The proposed hybrid artificial intelligence paradigm has been explored using a flexible manufacturing system (FMS) located in Eastern Mediterranean University laboratory (EMU- CIM lab) and the results are compared with Genetic Algorithm (GA) optimization technique, to successfully demonstrate the feasibility of the proposed architecture.

\section{Modern Manufacturing}

2.1. Internet of Things. Steady state industry has been changed to dynamic industry by recent industrial revolutions, which has resulted in manufacturers being pushed by the global market to reconsider their conventional manufacturing methods [26]. Modern manufacturing needs new manufacturing operations, and effective factory management has great value in this area [27]. Smart manufacturing is a powerful concept which can satisfy the needs of modern manufacturing by utilizing and adding high-tech products such as sensors, software, and wireless connectivity to required products [28]. Overall, utilizing high-tech equipment in manufacturing in order to optimize the manufacturing methods results in a new concept, which is known as the Internet of Things (IoT) [29].

The Internet of Things (IoT) can be defined as the interaction between technologies, which includes smart objects, machine-to-machine communication, radio frequency technologies, and a central hub of information, to monitor the status of physical objects, capturing meaningful data and communicating that information through IP networks and software applications (see Figure 1) [29].

In the IoT, to make objects detectable in order to monitor and collect the data required from them, they are equipped with an Auto-ID technology [30]. Utilizing this technology enables users to analyze the collected data, which can contain information such as temperature, changes in quantity, or other types of information, through wireless communication, and make efficient and accurate decisions [30].

In recent years with advances in technology, the IoT has started to adopt and utilize a new technology called Radio Frequency Identification (RFID), which is continuously increasing its market share, replacing traditional barcode technology and allowing the development of new applications [31].

RFID technology can be defined as a powerful innovative gadget which has been adopted in the development of the 


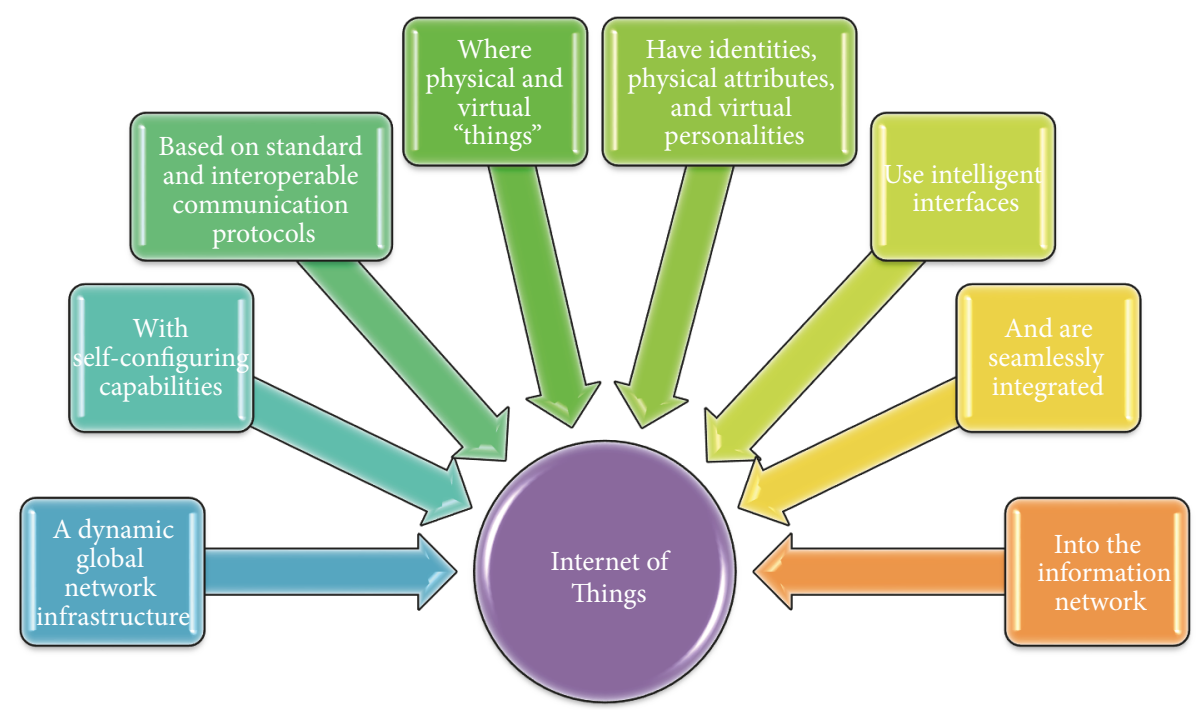

FIGURE 1: Definition of Internet of Things.

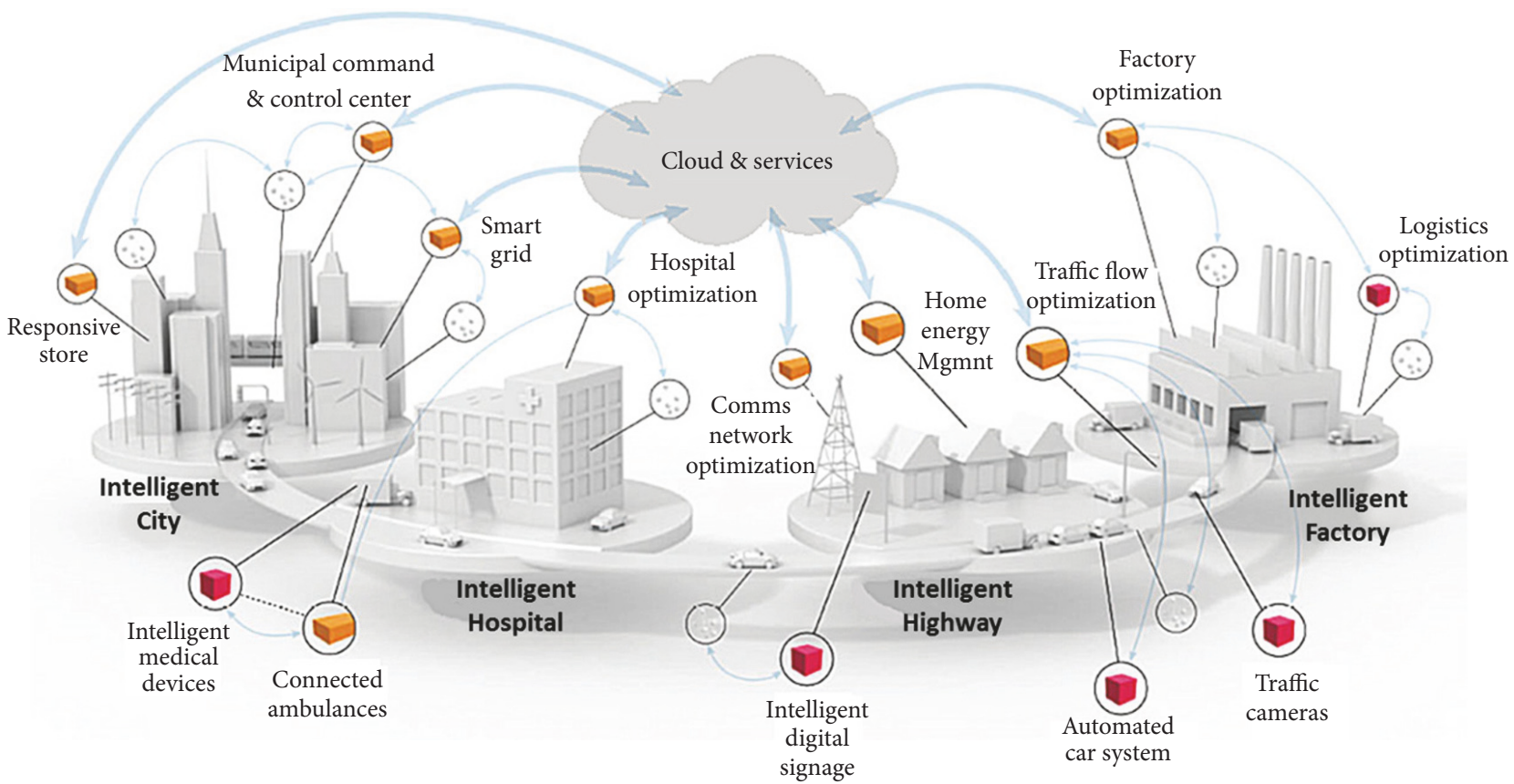

Figure 2: Internet of Things-Intelligent Systems Framework [24].

IoT [31]. RFID technology, as an application in the IoT, with accepted standards across industries, has been widely adopted in different manufacturing industry sections, such as supply chain management, smart manufacturing, home automation industry, and intelligent shopping (see Figure 2).

After giving a brief review about IoT and RFID technology in the next sections components and establishment of RFID technology have been introduced and discussed in detail.

2.2. Radio Frequency Identification Technology. Obtaining real-time information has a great value in different fields of the manufacturing industry such as flexible manufacturing systems, inventory management, and supply chain management. Recent advances in technology and modern industrial engineering systems, from production to transportation, have created a great need to track and identify the materials, products, and even live subjects [1]. One of the developing technologies which has been utilized as an application in the Internet of Things (IoT) to identify and track parts and objects in manufacturing industry is Radio Frequency Identification (RFID).

RFID technology is an Auto-ID technology which is adopted by the IoT as a reliable and efficient solution to the 


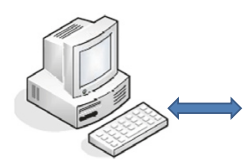

Host computer

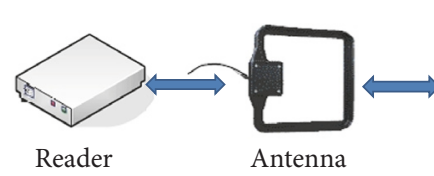

FIgure 3: Components of an RFID system.
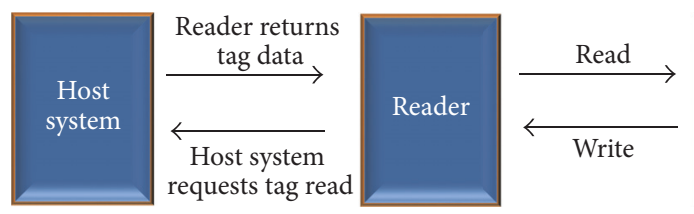

Tag

FIGURE 4: Interactions between components of RFID system. problem of object tracking and identification. RFID technology is known as an automatic identification technology as it uses wireless radio frequency waves, which are produced by an electromagnetic field, to transfer data to track and identify objects. Utilizing this technology enables users to analyze the collected data, which can contain information about the objects, such as temperature, changes in quantity, or other types of information, through wireless communication, and make efficient and accurate decisions [32].

It can be said that modern manufacturing has been changed by the utilization of Radio Frequency Identification. These changes are wide ranging and they include but are not limited to (1) the approach of tracking the objects in small and medium enterprises, (2) the boundaries of tracking the objects being upgraded to a global scale, and (3) interaction of the products with the production environment [32].

In the past, barcode technology had been used to track and identify objects and items in different manufacturing fields. While utilizing this technology was cheap, it should be mentioned that since collecting data had to be done manually by a barcode reader, it was not an easy and efficient task [33]. The other significant drawback of using barcodes is the fact that only the information about the object that was available at the time of reading could be stored; thus, barcodes do not provide the manufacturer required data in real time; they are only capable of recording data just in time [33]. Therefore, it is not an effective technology in modern manufacturing. Nowadays, this technology has been replaced with RFID technology which has been used effectively in industries and it has been a topic of research interest for many researchers. Gupta et al. [34] investigated RFID importance in production and operation management. Irani et al. [35] proposed a framework for presenting research obstacles related to RFID technology. A comprehensive research study about transferring real-time information using RFID technology in value adding chain components has been conducted by Huang et al. [36]. In general, this technology can be implemented in different fields, such as tracking and identifying patients in hospitals [2], warehouse item tracking [3], tracking pallets and cases in shipments [4], monitoring production lines [5], and supply chain management [6].

An RFID system is made up of two major parts: the hardware and the software. The hardware part consists of tags, readers, and antennas and the software includes middleware, which can be defined also as the computer unit [34]. It should be noted that the antenna can be part of the reader, meaning more than one antenna can be connected to one reader (see Figure 3).

RFID is an advanced technology which is utilized in data processing [37]. Required data from the objects are sent by tags to readers, antennas of readers receive these data, and they send it to a host computer as middleware to process it for further implementations (see Figure 4).

\section{RFID Network Planning (RNP)}

With advances in technology, the modern manufacturing industry is bounded with data interconnectivity. The key to being successful in the modern manufacturing industry is to obtain useful and in-time data, which can be part of the tracking devices used in the supply chain management, which are used to track and map a live subject or parts of the flexible manufacturing industry, to provide alerts to manufacturers in regard to the need for maintenance of parts [37]. Manufacturers need to collect required data for further analyses by deploying different types of gadgets of the IoT [38], such as sensors, network cameras, or RFID systems, in their operational field [38].

An RFID system, as discussed in this paper, includes four major elements: tags, readers, antennas, and a computer unit. It should be remembered that the antenna, as an internal antenna, can be part of the reader, or it can be mounted as an external antenna. Also, each reader can adopt one or more than one antenna. Readers, through the use of antennas, collect the data sent by tags and they relay this to a host computer to process it for further implementations. In essence, RFID is the technology of processing data, which is comprised of radio frequency signals emitted from mounted tags on subjects that are sent by readers to a host computer unit [26].

There are three types of tags: passive, semipassive, and active. The differences between these is their source of power; active and semipassive tags are battery powered but passive tags do not have internal power [27]. It should be noted that, in this paper passive tags, because they have advantages such as being cost efficient and having a long life cycle as compared to that of semipassive and active tags, have been adopted in the RNP [28].

The data of a tag can be read by a reader across a certain distance between the tag and antenna of the reader. It means that a reader through its antenna can receive the information of a tag within a limited range. The establishment of communication between the antenna and tag thus relies on the distance between the tag and antenna, and it is highly sensitive to changing distances [29]. Because of the distance problem, there is a possibility of not being able to read the data of a tag by using a reader; this is termed as the "uncovered tag problem."

Utilizing more antennas is a common solution which has been adopted in recent years to overcome this problem [39]. 


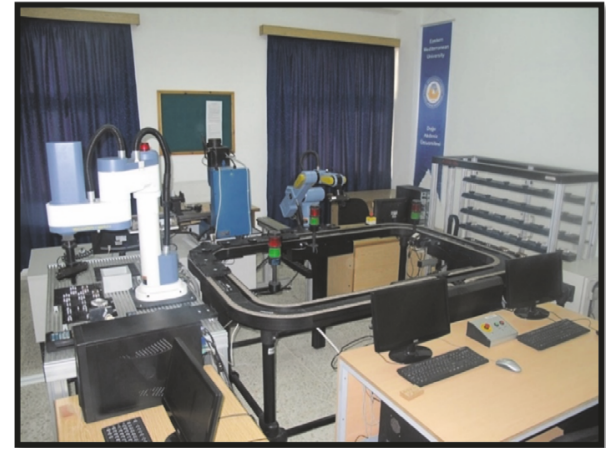

FIGURE 5: FMS laboratory of Eastern Mediterranean University (EMU).

By adopting more antennas in the RFID system, important criteria such as number of antennas and positions of them, collision of the antennas, and coverage of the network need to be calculated. Answering such questions has led to an important concept known as RFID Network Planning (RNP) $[13,23]$.

3.1. Mathematical Modelling of RFID Network Planning. To model an RFID network mathematically some important criteria such as number of tags, number of antennas, coverage percentage of the network, collision percentage of antennas, and transmitted power in the network should be considered. One of the most reliable mathematical models which has been adopted by many researches to deal with RNP [13, 40, 41] is the Friis transmission equation [42]. In this paper, a developed model of this equation which has been proposed by Gong at el. [13] is utilized to deal with RNP.

The first step in modelling a RFID network and in solving a RNP is defining the working area, after which follow defining the number of tags which are needed to be read by readers and, finally, defining the number of antennas of the readers.

3.1.1. Working Area. The first step of designing a RFID network is defining the working area across which RFID tags should be covered by deployed antennas, and the proposed hybrid artificial intelligence algorithm has to be implemented to this working area to optimize the number and positions of the deployed RFID antennas. To perform this task, a dynamic working area has been proposed in this paper.

The dynamic state refers to the movement state of the tags which has been termed as "moving tags." Contrary to the static working area, in which tags have been pinned to their locations, in the dynamic working area, tags have changing positions and they have a moving status.

The proposed dynamic working area in this paper is the conveyor belt located in the Flexible Manufacturing Systems (FMS) Laboratory of Eastern Mediterranean University (EMU) (see Figure 5).

Using MATLAB software, the conveyor belt, which has $3 \mathrm{~m}$ length and $2 \mathrm{~m}$ width, has been modelled. It is assumed that the conveyor moves with constant speed of $0.3 \mathrm{~m} / \mathrm{s}$, and

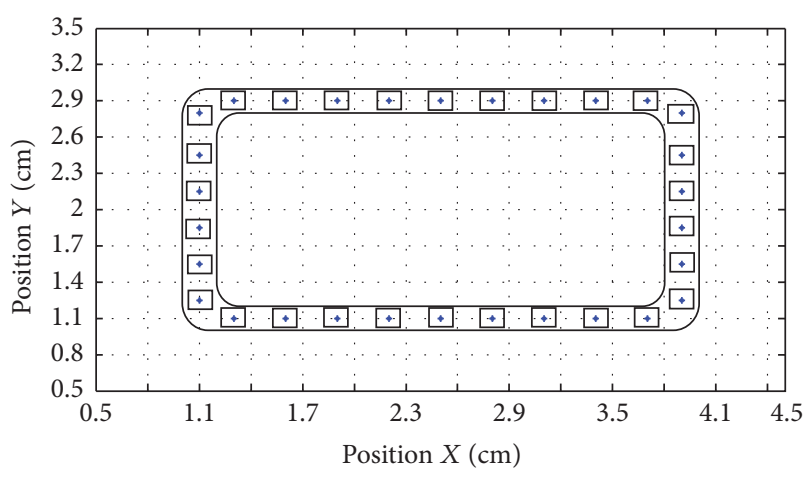

+ Parts with mounted tags

FIGURE 6: Proposed dynamic working area.

square shape parts with length of $15 \mathrm{~cm}$, which have been equipped with RFID passive tags, move along the conveyor with $15 \mathrm{~cm}$ distance between each other (see Figure 6).

The tags are mounted on the middle of each part and they move with the parts, so, by definition, the proposed working area conditions can be interpreted in the following two different scenarios:

(i) One tag moves along the conveyor; its speed is equal to conveyor speed and, every 2 seconds, the tag moves $30 \mathrm{~cm}$; thus, in order to complete a tour of the conveyor belt, the tag should be detectable in 30 different positions.

(ii) Tags move along the conveyor and their speeds are equal to conveyor speed, so, every second, each tag moves $30 \mathrm{~cm}$, so, on whole conveyor belt, 30 tags move at the same time.

It should be noted that all 30 tags should be covered by RFID antennas at the same time, so it can be interpreted that 30 static tags have been deployed on the RFID network in both cases. After defining the working area and modelling it with MATLAB software, the next step is to define the parameters of the proposed RNP.

3.1.2. Coverage. Coverage of the network is defined as the percentage of the tags which have been read by the readers via the antennas of the readers [13]; the most important criteria which should be satisfied in RNP is achieving $100 \%$ coverage of tags.

As mentioned before, the distance between the tag and antenna plays a major role in tag coverage. Also, passive tags do not use a power source for transmitting RF signals; they reflect back the emitted power provided by the antennas of readers. The internal circuit of a passive tag is powered up (activated) by a RF signal (an electromagnetic wave), which has a greater power than the threshold of the tag. The powered up tag then starts to reflect back the signal to the antenna of a reader and if this signal has been received by an antenna, it can thus be said that communication between the tag and reader through the antenna has been established and 
that tag has been covered [47]. This entire procedure can be summarized using the following formula [13]:

$$
\begin{aligned}
& C_{v}(t) \\
& \quad= \begin{cases}1, & \text { if } \exists a_{1}, a_{2} \in A S, P T_{a_{1}, t} \geq T_{t} \wedge P A_{t, a_{2}} \geq T_{a} \\
0, & \text { otherwise. }\end{cases}
\end{aligned}
$$

$C_{v}(t)$ is the calculated coverage of each single tag. The adopted formula for $N$ tags is defined as below [13]:

$$
\mathrm{COV}=\sum_{t \in T S} \frac{C_{v}(t)}{N_{t}} \times 100 \% .
$$

$\mathrm{COV}$ is the calculated total coverage of the network for the all tags.

The power received by the tags $P T_{a, t}$ and the power transmitted by the tags, $P A_{t, a}$, are calculated using the Friis transmission equation, as shown below [13]:

$$
\begin{aligned}
P T_{a, t}[\mathrm{dBm}]= & P_{1}[\mathrm{dBm}]+G_{a}[\mathrm{dBi}]+G_{t}[\mathrm{dBi}] \\
& -L[\mathrm{~dB}], \\
L[\mathrm{~dB}]= & 10 \log \left[\left(\frac{4 \pi}{\lambda}\right)^{2} d^{n}\right]+\delta[\mathrm{dB}], \\
P A_{t, a}[\mathrm{dBm}]= & P_{b}[\mathrm{dBm}]+G_{t}[\mathrm{dBi}]+G_{a}[\mathrm{dBi}] \\
& -20 \log \left(\frac{4 \pi d}{\lambda}\right), \\
P_{b}= & \left(\Gamma_{\text {tag }}\right)^{2} \times p_{t},
\end{aligned}
$$

where $P_{1}$ is the transmitted power of the antenna, $G_{a}$ is the gain of the antenna, $G_{t}$ is the gain of the tag, $L$ represent loss, $\lambda$ is the wavelength, $d$ is the distance between the tag and antenna, and $n$ depends on the environment and it varies from 1.5 to $4 ; \delta$ represents other losses and $P_{b}$ is the backscatter power transmitted by the tag which is reduced by multiplying it by the reflection coefficient $\Gamma_{\text {tag }}[13]$.

3.1.3. The Number of Antennas. Deploying each antenna to a network has a cost. It is possible that elimination of a few deployed antennas in an RFID network has no effect on the network coverage; as a result, they are redundant and inefficient. These antennas impose an unnecessary cost to the network, and, thus, it is an important criterion in the RNP to calculate the most efficient number of antennas. The mathematical representation of this concept is shown below [13]:

$$
N_{a}=N_{\text {max }}-N_{\text {red }},
$$

where $N_{\max }$ is the maximum number of antennas that are deployable in the network, which is calculated through performing an optimization algorithm in each step. However, it should be noted that for the first step it should be taken as a random number; in this paper, it is assumed as 20 which is equal to the number of tags. $N_{\text {red }}$ is the number of redundant antennas and $N_{a}$ is the efficiency of the antennas.
3.1.4. Interference. Interference is another important criterion in RNP which can be defined as collision between the efficient antennas [13]. Collision is a type of interference in the network and it occurs as a result of the interrogation of one tag by more than one antenna of the readers. The equation shown below is a mathematical representation of a collision which is accrued by one tag [13]:

$$
\gamma(t)=\sum P T_{a, t}-\max \left\{P T_{a, t}\right\}, \quad a \in A S \wedge P T_{a, t} \geq T_{t} .
$$

The total interference of the network can be calculated by summing all of the collisions of the network. The mathematical representation of the total interference of the network is shown as below [13]:

$$
\mathrm{ITF}=\sum_{t \in T S} \gamma(t)
$$

3.1.5. The Sum of the Transmitted Power. As noted before, the distance between the tag and antennas has a major effect on network coverage. Since passive tags have been used in this paper to model the RNP network and since passive tags will be activated by absorbing the transmitted signal of the antennas of readers, the transmitted power of each antenna thus has a direct relationship with its interrogation range. This reduces the amount of transmitted power which may result in a decrease of coverage of the network. Its lowest criterion in RNP is given by [13]

$$
\mathrm{POW}=\sum_{a \in A S} P S_{a}
$$

where $P S_{a}$ is the amount of power transmitted by antennas.

After introduction to the RFID Network Planning, criteria which are important and should be calculated in RNP have been introduced. It should be noted that mathematical representation of RNP is not capable of making changes in the coverage, interference, or redundancy of the network, by changing the number of deployed antennas, so, in the next section, a novel artificial intelligence technique has been proposed to satisfy these criteria.

\section{Hybrid Artificial Intelligence Optimization Technique}

In brief, the goal of RNP and its optimization can be summarized by the following statement: "to plan a cost efficient RFID network it is necessary to minimize the number of antennas, minimize interference of antennas and maximize coverage area of objects" $[11,16]$.

Therefore, in this paper, to satisfy these targets and to design an efficient RFID network, a hybrid optimization technique is introduced. The "hybrid" term refers to combining different algorithms to optimize the RNP as one optimization approach (see Figure 7).

For this reason, Ring Probabilistic Logic Neural Networks (RPLNN), a novel technique in RNP optimization, has been used. The algorithm of RPLNN is designed in a way which enables the paradigm to adjust the number of embedded antennas in the network; this makes the RPLNN optimization 


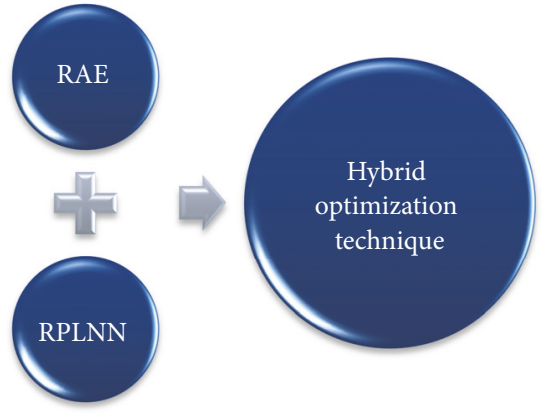

FIgURE 7: Proposed hybrid optimization technique.

technique an efficient artificial computational intelligent optimization approach, in order to deal with complex RFID Network Planning problems.

The second component of the proposed hybrid algorithm is utilizing the Redundant Antenna Elimination (RAE) optimization technique in addition to the RPLNN optimization technique. Utilizing the RAE algorithm has two advantages: the first is reducing optimization process by reduction of iterations, and the other one is to give flexibility to the RNP solution in terms of number of antennas.

The first step of the RNP optimization process starts with solving the proposed mathematical model of RNP, which was discussed in the previous section of this paper (see Figure 8). All parameters of RNP, which are coverage, redundancy, and interference of the RFID network, should be calculated. To fulfil this task, the total number of deployed RFID antennas in the network should be known; thus, in the first iteration, this number has an arbitrary value.

After calculating all parameters of RNP, the next step is the optimization process, which is done by using the Redundant Antenna Elimination (RAE) algorithm. In this step of the proposed hybrid artificial intelligence algorithm, all deployed antennas which are nonefficient antennas and impose extra cost to the network, as a result of redundancy, are eliminated and deleted from the RFID network. After the RAE optimization process has been finalized, the total number of nonredundant antennas which remain on the RFID network is calculated.

The next step of the optimization process is to apply the second phase of the hybrid artificial intelligence algorithm; the Ring Probabilistic Logic Neural Networks (RPLNN) algorithm. It is important to know that the RPLNN algorithm optimizes the RNP based on nonredundant antennas, which have been determined by the RAE algorithm.

Following this, it should be checked whether the optimization process has been finished or not. In order to perform this task, firstly the number of iterations, which are required for whole hybrid optimization process to optimize RNP, should be defined by the user. After optimizing RNP utilizing the RPLNN technique, if the iterations have reached the predefined number, then the optimization process should be stopped and the best solution for RNP should be chosen. If the iterations have not been completed, then the maximum number of deployed antennas in the network should be calculated and RNP optimization process should be continued from the step of solving the mathematical model for RNP. The hybrid artificial intelligence optimization process continues until the iterations reach the predefined number of iterations.

An introduction to the proposed hybrid artificial intelligence algorithms and an overview flowchart of the algorithm have now been provided; thus, the entire optimization process has been introduced. In the next section, the two components of the hybrid artificial intelligence algorithm, which has been adopted to deal with RNP problem, are introduced. These algorithms, which are termed in this paper as "Redundant Antenna Elimination algorithm" and "ring probabilistic logic neural networks," have been discussed in detail in the following sections.

4.1. Redundant Antenna Elimination Algorithm. The logic of the optimization process through using the Redundant Antenna Elimination (RAE) paradigm is based on removing redundancy from the RFID network. The terms"redundancy" means that an existing RFID tag has been covered by two different RFID antennas of readers. It happens when a tag receives an activation signal from an antenna and starts to backscatter this signal; the emitted signal from the tag can be received by more than one antenna and, thus, the tag will be covered by more than one antenna. Since coverage of the network by eliminating all of the antennas except one will not be changed, so it means that these antennas can be considered as redundant antennas on the network and should consequently be eliminated. In the first step of the optimization process of RAE algorithm which optimizes RNP, each iteration of the optimization task works by eliminating redundant deployed antennas using the following procedure.

Firstly, coverage of the RFID network is calculated using the RNP mathematical model, after which the redundancy of the network is calculated using the mathematical formulation, which has been previously discussed. To perform this task, deployed antennas in the RFID network should be eliminated one by one and coverage of the RFID network should be calculated after each antenna elimination. If the total calculated coverage of the RFID network after each antenna elimination remains the same as before, then the eliminated antenna remains as deleted. Otherwise, if the total calculated coverage of the network after the antenna elimination is reduced, the RFID network coverage before the antenna elimination should be recovered and be undeleted. This procedure should be repeated for all of the antennas.

Each calculation step which has been performed for each antenna comprises one iteration, so the number of the iterations of this algorithm should be equal to total number of deployed antennas in the RFID network. In each iteration of the optimization process utilizing the RAE paradigm, if a redundant antenna has been found by the algorithm, the case number of the redundant antennas which have been eliminated will be increased by one.

After performing the last iteration of the RAE optimization process, which is an investigation of the redundancy of the last deployed antenna in the RFID network, the total number of redundant antennas can be calculated. 


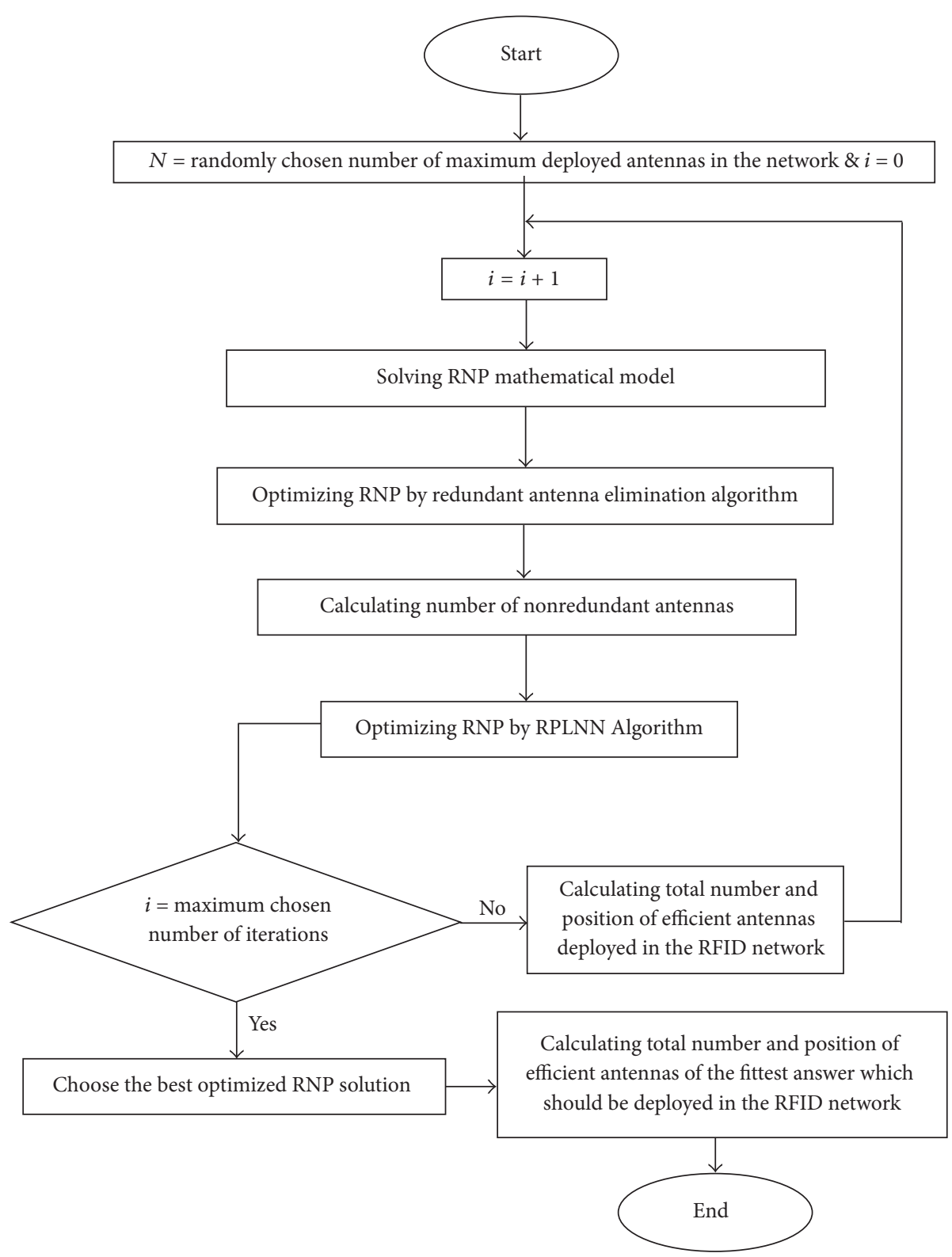

FIGURE 8: Proposed hybrid artificial intelligence optimization process.

By calculating this number and by knowing the total number of deployed antennas in the RFID network, the total nonredundant antennas can thus be calculated. Therefore, the next step of the hybrid optimization technique, which is performing optimization through using the RPLNN paradigm on nonredundant antennas, can be performed. The proposed RAE optimization algorithm can be summarized as shown in the following steps (see Figure 9):

(i) Total coverage of RFID network should be calculated $\left(C_{1}\right)$.

(ii) One of the deployed antennas in the RFID network should be eliminated.

(iii) After the antenna elimination, again the total coverage of RFID network should be calculated $\left(C_{2}\right)$. (iv) If coverage of the network after antenna elimination is calculated as less than before the antenna elimination $\left(C_{2}<C_{1}\right)$, then the eliminated antenna should be recovered.

(v) If coverage of the network after antenna elimination is calculated as equal to before the antenna elimination $\left(C_{2}=C_{1}\right)$, then the antenna should not be recovered and the number of the redundant antennas should be increased by one.

(vi) The RAE optimization process will be finished after investigating the redundancy status of the all deployed antennas in the network, after which the number of the nonredundant antennas will be calculated. 


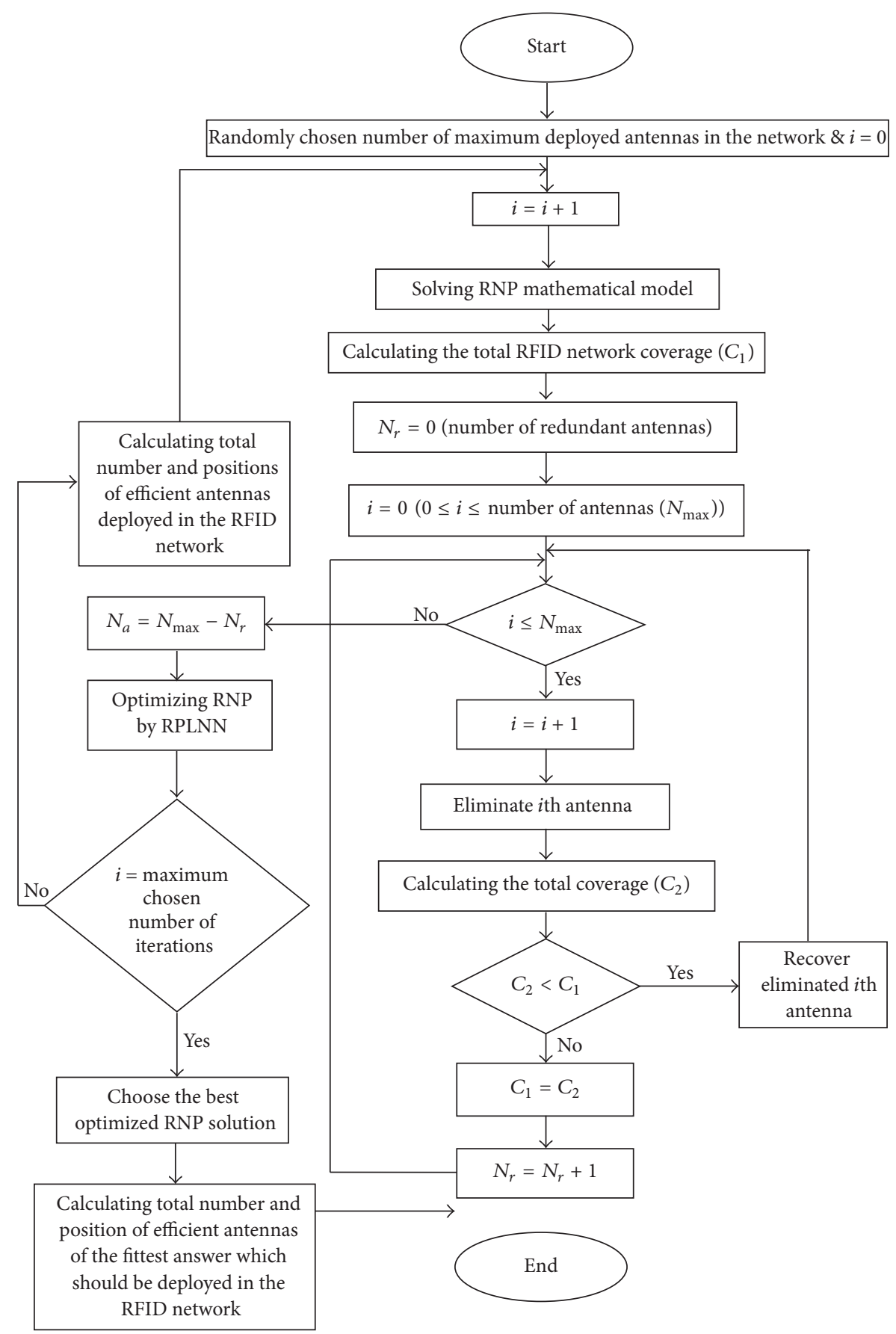

Figure 9: Flowchart of the proposed Redundant Antenna Elimination algorithm.

(vii) The system is now ready for the next phase of the hybrid artificial intelligence optimization process.

4.2. Ring Probabilistic Logic Neural Networks. The Probabilistic Logic Neuron (PLN) is a RAM based device which was proposed in 1988 by Kan and Aleksander [48]. As seen in Figure 10, a PLN is made up from a probabilistic node based on the input array which is used to calculate the output array. The term "probabilistic node" refers to the probability percentage of each node; this can be calculated by dividing the output of a PLN by the maximum output, which can be stored in memory.

PLNs, in regard to their value and length of the output, have two major differences as compared to RAM neurons. RAM neurons can only store their output with a length of one bit in the memory location; however, PLNs are capable of storing outputs which have greater lengths than one bit; these are known as "B-bit." The other difference is that the outputs of the RAM neurons can be 0 or 1 ; however, the outputs of PLNs have an additional third state which is called the 


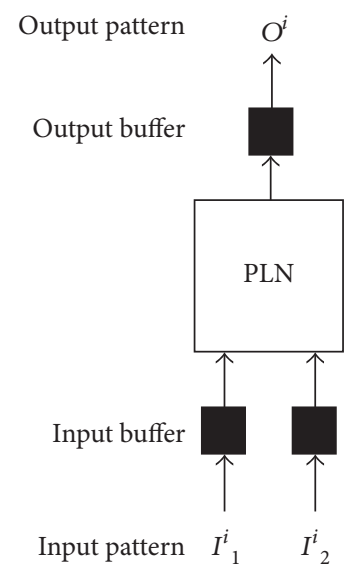

Figure 10: Probabilistic Logic Neuron (PLN) [25].

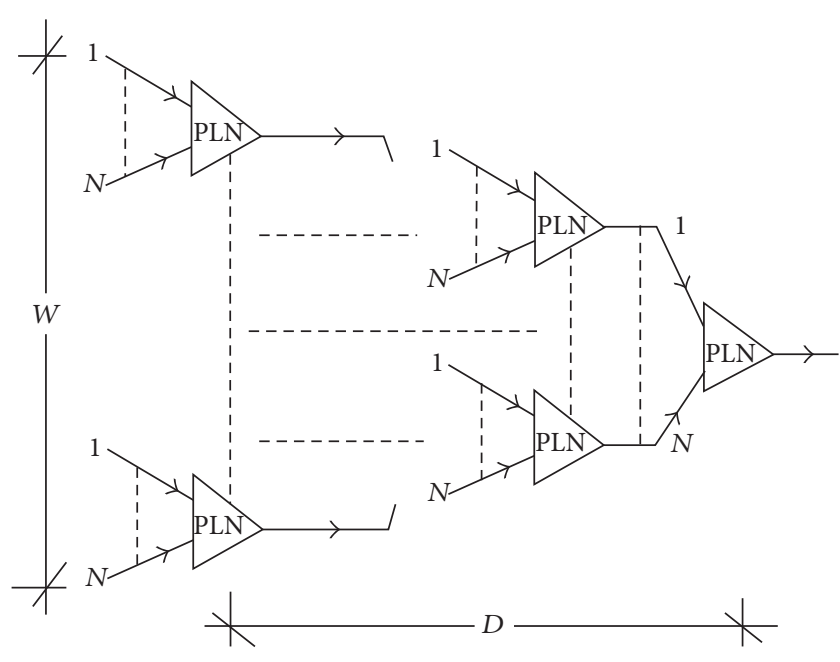

Figure 11: Pyramidal PLN Neural Network structure [43].

"Don't Care" state and it is represented by "X"; the "Don't Care" state refers to the outputs which can have a value of 0 or 1 , both with a probability of $50 \%$.

By adopting the "Don't Care" state, the meaning of the output 0 has been changed in PLNs. It means that the state of 0 has two meanings in the PLN concept. Firstly, it can be interpreted that, for the given input vector, the PLN neuron has not been trained, and, secondly, the 0 can be interpreted as the calculated output of the trained PLN, which is the opposite of the RAM neuron concept. It can be said that utilizing this third state enables PLNs to have a better performance than RAM neurons. Another important point to note is that they can have more than two layers, which RAM neurons cannot have. As seen in Figure 11, PLNs can be combined together and built into a PLN Neural Network with a paramedical structure.

It has been proven that the PLN Neural Networks can deal with hard learning problems [43] and consist of many PLN neurons (shown as size of $W$ ), that each has $N$ inputs and more than one hidden layer (shown as size of $D$ ), ensuring the robustness of PLN Neural Networks.
In general, PLN Neural Networks do not have a rigid structure; based on the requirements of the problem, by combining PLNs in different manner, you can design different PLN Neural Networks (see Figures 11 and 12).

These kinds of PLN Neural Networks are also known as Multilayer PLN (MPLN) networks. The efficiency of MPLN networks has been investigated and analyzed in detail by Zeng et al. [49]. Their research result indicates that flexibility in arrangements of PLNs provides an advantage of having fast and efficient convergence times to find the final solution of hard learning problems, through the use of MPLNs.

In 2002, based on the flexibility of the structural design of MPLNs, a new structure, which is known as "Ring Probabilistic Logic Neural Networks" (RPLNN), was proposed by Menhaj and Seifipour [50] to deal with optimization problems. The proposed structure connects the input of the first PLN to the output of the last PLN; this connection is based on the available data sets. These can be divided into two different groups, which are called "feed forward" and "feedback connections."

The feed forward connection state happens when the input data of the first PLN becomes available; this data is sent as the feed forward signal to the output of the last PLN. The feedback connection state happens when the output data of the last PLN becomes available; this data is sent as the feedback signal to the input of the first PLN. This feedback and feed forward connection line, which connects the first and last PLNs, is known as the "ring structure," and because of this property this kind of MPLN network is known as "Ring Probabilistic Logic Neural Network” (RPLNN). In 2016, Azizi et al. [45] utilized the RPLNN structure as part of a weightless Neural Network to optimize a weighted Artificial Neural Network model of the mechanical behaviour of the friction stir welding process (see Figure 13). A special structure of this algorithm, in which the inputs and outputs of the RPLNN have been defined, has been implemented by Azizi et al. [44] to deal with the static RNP problem and the same model has been used to optimize the proposed dynamic RNP model in this paper.

In RPLNN structure each PLN has its own truth table which sets the output value based on the input vector of either the 0,1 , or Don't Care states. The training of the RPLNN can be summarized as continuing the training process until all Don't Care states have been replaced with values of either 0 or 1 [51]. It means that the RPLNN structure, which is the second phase of the proposed hybrid artificial intelligence optimization paradigm, fulfils the task of optimization, based on pure random search among all of the possible solutions $[52,53]$.

The RPLNN optimization process starts with converting the inputs into binary codes, which are made of zeroes and ones. This step is followed by creating a population of possible answers to the problem, after which each of the possible answers in the defined population is evaluated in order to know which of them is the best answer and which one has the best value; performing the evaluation task by defining a fitness function is essential. It should be noted that the fitness function should be defined using the criteria of the problem; these need to be optimized and they differ from one problem 


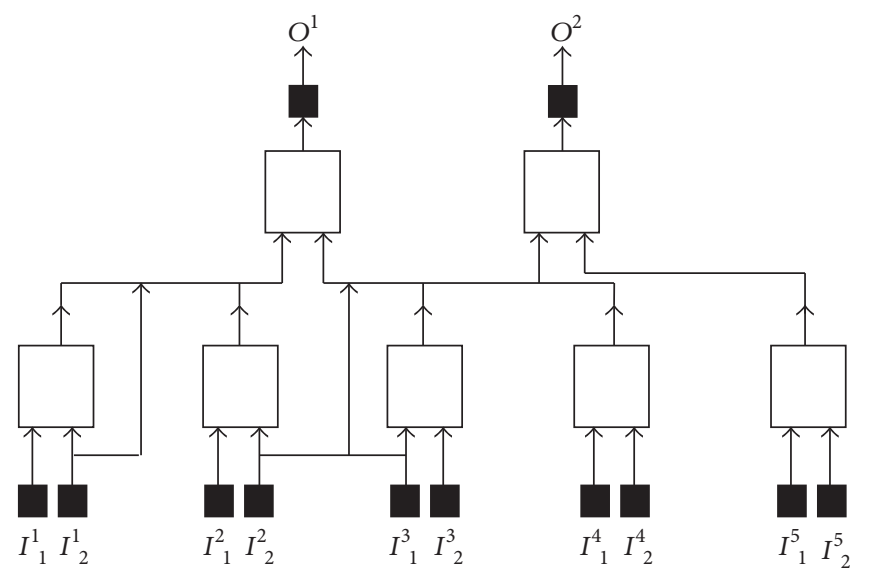

FIgURE 12: Example of a PLN Neural Network structure [44].

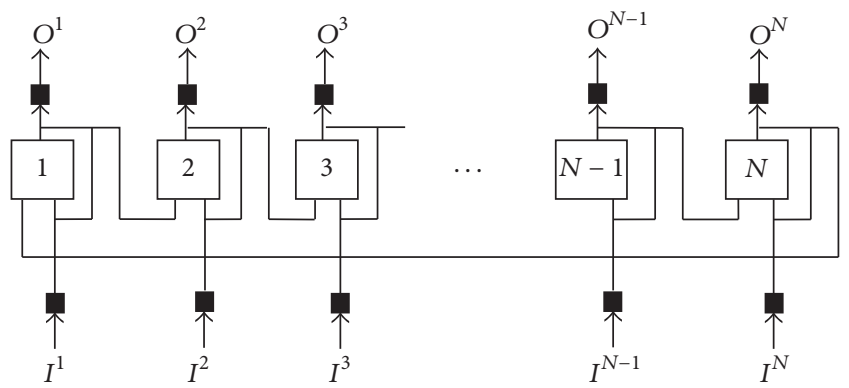

FIgURE 13: RPLNN structure [45].

to another. The fitness function which has been used in this paper to optimize RNP is introduced in detail in the next section. The proposed RPLNN algorithm which has been adopted in this paper is part of the proposed hybrid artificial intelligent algorithm, as shown in the flowchart in Figure 14.

After calculating the total number and position of nonredundant antennas using the first part of the proposed hybrid optimization paradigm, which is RAE, the second phase of the optimization is begun with RPLNN; all criteria of RNP mathematical model are calculated by utilizing the positions of nonredundant antennas. The calculated positions of nonredundant antennas in the parallel procedure are encoded as binary code to create the population of all possible answers. The detailed discussion about how to create the population of all possible answers is provided in the next section. Each possible answer in the population is termed as "individual," and the next step required is to evaluate each of these individual answers by using the defined fitness function to optimize RNP, which is discussed in detail in the next section.

At the same time, the parallel process output of each PLN in the proposed RPLNN structure should be calculated by using the PLN truth table to encode the binary positions of nonredundant antennas as inputs. Next, the calculated binary output should be decoded to decimal vectors, which are the new positions of antennas, and, consequently, the RNP mathematical model based on these new positions of antennas should be solved.

The next step is to calculate the fitness function of RNP based on calculated criteria, which are obtained by applying the new positions of the antennas. Following this, a comparison should be made between two calculated fitness functions based on two different positions of antennas, given as RPLNN inputs and outputs. If the fitness function of the RPLNN output is less than the calculated fitness function of the RPLNN input then all calculated RPLNN outputs, which are the binary form of positions of antennas, should be returned back to the "Don't Care" status; otherwise, all calculated outputs should be saved. These processes continue until the optimization process completes all of the predefined number of iterations. The final step is to choose the best answer of the calculated population of the antennas based on its fitness function. It means that the fittest individual of the population of answers is the best solution of the proposed optimization algorithm.

The second phase of the proposed hybrid artificial intelligence algorithm, RPLNN, which has been adopted to deal with RNP in this paper, can be summarized as the following steps:

(1)

(A) Encode the positions of nonredundant antennas calculated by RAE to binary codes and create population of possible answers.

(B) Solve RNP for calculated nonredundant positions of antennas by RAE.

(A) Calculate the output of each PLN of RPLNN by using truth table of PLN, in regard to inputs.

(B) Decode RPLNN outputs to decimal position of antennas vectors.

(C) Solve RNP for calculated positions of antennas. 


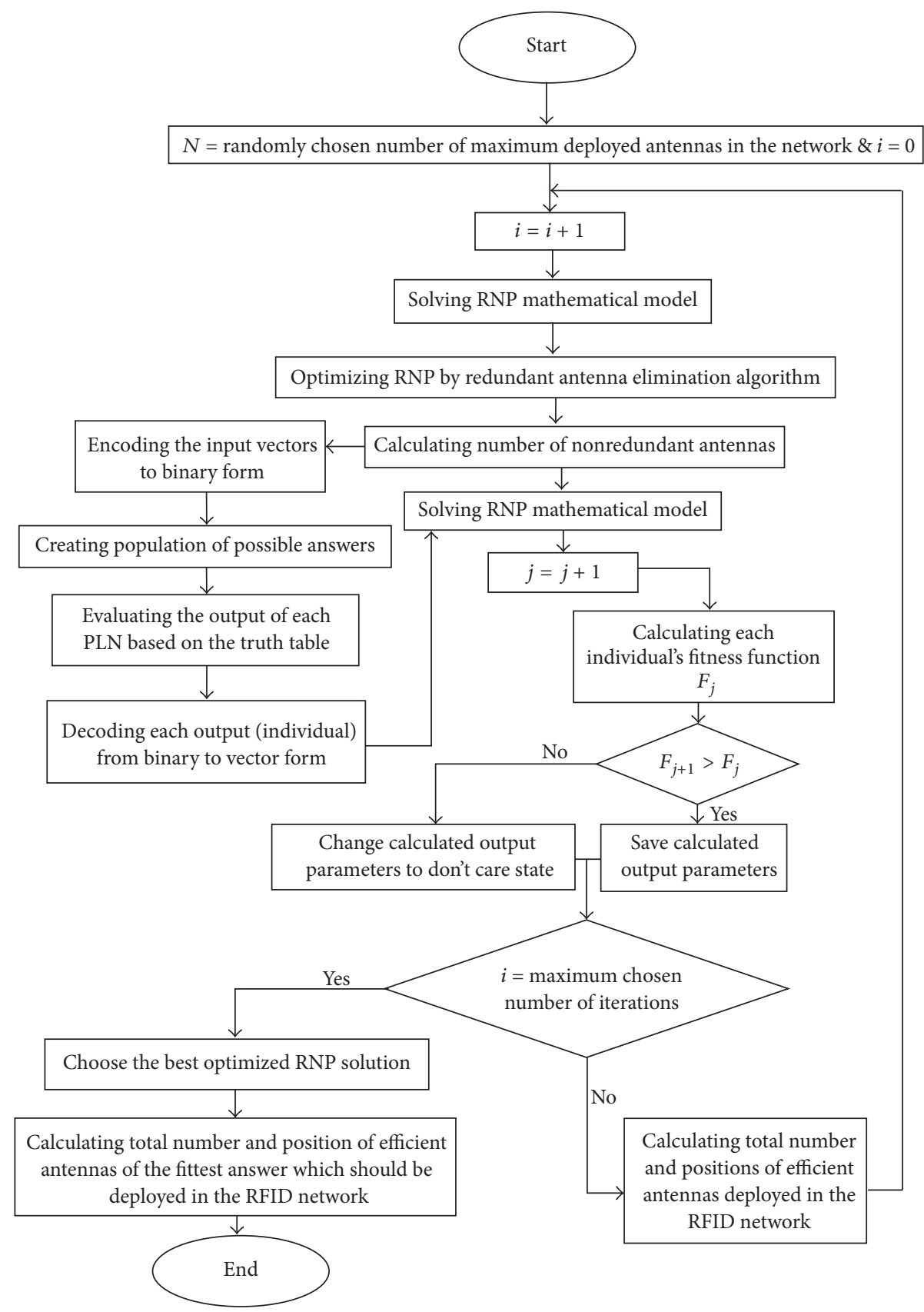

FIGURE 14: Flowchart of the proposed RPLNN algorithm to optimize RNP.

(3)

(A) Calculate the fitness function value for each individual input of RPLNN, which are calculated positions of antennas.

(B) Calculate the fitness function value for each individual output of RPLNN, which are calculated positions of antennas.

(4)

(A) If the calculated fitness function value of the output of RPLNN is more than the calculated input fitness function value, then save the value of the outputs of the RPLNN.

(B) If the calculated fitness function value of output of RPLNN is less than or equal to the calculated input fitness function value, then reset the value of the outputs of the RPLNN and set all of them as the "Don't Care" state.

(C) Repeat these steps for the predefined number of iterations.

(5) Choose the best and fittest answer out of all possible solutions. 
(6) Calculate number and positions of efficient antennas which should be deployed on RFID network.

\section{Parameters of the Proposed Hybrid Algorithm}

The parameters of the proposed hybrid artificial intelligence algorithm which should be defined are divided into two sections: firstly, the population of the possible answers should be defined and, secondly, the objective function of the algorithm should be defined.

5.1. Population of the Possible Answers. The population of the possible answers can be defined as a matrix with $m$ rows and $n$ columns, in which each row of the matrix contains a possible answer for optimizing the proposed RFID network. The number of rows is a predefined arbitrary number which has been assumed in this paper as 100; thus, the optimization process starts with 100 possible answers to RNP known as the global answer space or population of the possible answers; it tries to help the optimization process to be accomplished by enabling the best answer in the global answer space to be searched for.

As discussed in the previous sections, the optimization process starts with solving the mathematical model of the
RFID network and it ends with choosing the best solution. Solving the mathematical model needs the number and positions of the RFID antennas, which in the first iteration of the proposed optimization technique has been chosen randomly (here, in this paper, it has been chosen to be the number of the tags). However, in the other iterations, the required data has been provided by the RPLNN algorithm. Each answer in the population of possible answers can be defined as the combination of the position of deployed RFID antennas and the activity status of the antennas.

The positions of the antennas have been defined using Cartesian coordinates as $(x, y)$, and the activity status refers to the state of the antenna, which is on or off, so if an antenna activity is calculated as off, it means that the antenna has not been deployed on the RFID network.

The positions and activity statuses of the antennas should be encoded in binary form as zeroes and ones at the output of the RAE algorithms, which is the input of the RPLNN algorithm, and they should be decoded to decimal form at the input of the mathematical model, which is the output of the RPLNN algorithm. In this case, the number of the columns of the population matrix is equal to the binary string length of the positions and activity statuses of the antennas (see (8)).

\section{Proposed Population of Possible Answers}

$$
\left[\begin{array}{c}
x \text { positions of antennas } y \text { positions of antennas activity of antennas } \\
x \text { positions of antennas } y \text { positions of antennas activity of antennas } \\
x \text { positions of antennas } y \text { positions of antennas activity of antennas } \\
\vdots
\end{array}\right] \text {. }
$$

It should be noted that, for the activity statuses of the antennas in this paper, 0 has been assigned for nonactive antennas and 1 has been assigned for active antennas. An example of the possible answer encoded to binary form has been illustrated in Figure 15.

5.2. Fitness Function. The fitness function is an evaluation tool which rules the optimization process, and it can be interpreted as a combination of the parameters of the RNP, which should be maximized or minimized through using the optimization algorithm. Each row of the population of the possible answers matrix should be evaluated by the fitness function, and the possible answer which has the highest value of the fitness function is the best optimization solution of the RNP.

The aim of the proposed hybrid artificial intelligence algorithm in this paper is the establishment of an optimized RFID network by minimizing the number of deployed antennas, in a manner which maximizes the coverage percentage of the network and which reduced the interference of the network to the lowest possible value.
The following fitness function which has been proposed in this paper to evaluate the performance of each possible answer of population matrix is

$$
\begin{gathered}
f\left(x_{i}\right)=\frac{100}{1+(100-\operatorname{COV}(i))^{2}}+\frac{100}{1+\operatorname{ITF}^{2}(i)} \\
+\frac{1}{1+(\text { Number of Antennas })^{2}} \\
\quad i=1, \ldots, \text { population size. }
\end{gathered}
$$

The value of the proposed fitness function increases by increasing the network coverage and by decreasing the interference and the number of deployed antennas in the network, since coverage of the network depends on the RFID antennas; that is, tags should be covered by at least one antenna deployed on the network. In this case, if the network coverage by adopting one antenna reaches $100 \%$ and interference reduces to zero, the maximum value of the proposed fitness function value will be calculated as 201 .

After defining the working area and parameters of the proposed hybrid algorithm in the next part, the section of the 


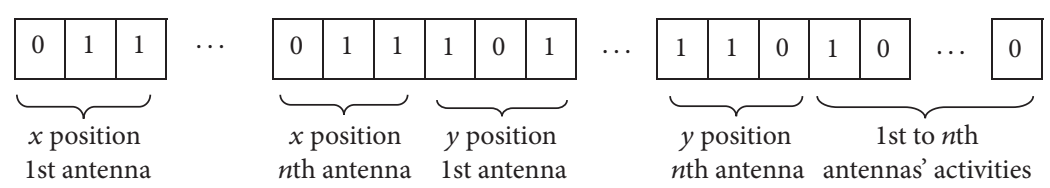

FIGURE 15: Example of encoded answer in population of answers.

paper which follows this presents the results of optimizing the RNP by using the proposed algorithm.

The Genetic Algorithm (GA) is one of the most wellknown evolutionary optimization techniques, which has been adopted by many researchers to optimize complex problems [54,55]. Briefly, the optimization process by GA can be divided into 6 steps, which are given as follows [46]:

(1) Create population of possible answers.

(2) Evaluate fitness function.

(3) Create next generation of possible answers.

(4) Apply crossover.

(5) Apply mutation.

(6) Repeat Steps (2)-(5).

The first and the second steps, which are creating the population of possible answers and evaluating the performance of them by using the fitness function, respectively, have the same procedure as has been discussed in the previous section of this paper.

The next step is creating the next generation of the population of possible answers by adopting an appropriate selection procedure $[56,57]$. In this paper, the roulette wheel selection approach [46] has been utilized to select the best answer through calculating the fractional fitness function of each possible answer, which has been defined as below:

$$
F\left(x_{i}\right)=\frac{f\left(x_{i}\right)}{\sum_{i=1}^{n} f\left(x_{i}\right)}
$$

$i=1, \ldots$, number of antennas.

The number of the possible answers of the population has been taken as 100 in this paper; thus, the roulette wheel is spun 100 times to select an answer to generate the next generation of possible answers (see Figure 16).

The next step is to apply the crossover to the generated population. The crossover operator combines different parts of two different answers (chromosomes) known as parents, and it produces new chromosomes known as children. The crossover can be adopted as either a twopoint crossover (see Figure 17(a)) or a single point crossover (see Figure 17(b)) [46]. In this paper, a single point crossover has been adopted as the operator of GA.

The final step of optimization by GA is adopting another operator known as mutation. The mutation operates as the NAN function on one of the binary bits of the answers (gene). It means that if the gene has a binary value of 0 , the mutation operator will change it to 1 , and if it has a binary value of 1 , the operator will change it to 0 (see Figure 18).

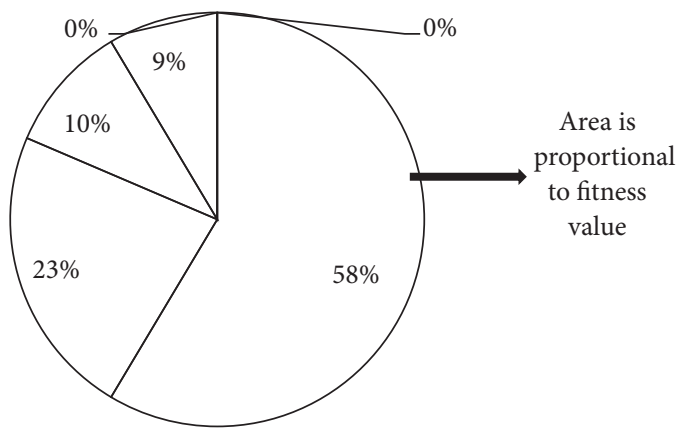

FIgURE 16: Example of Roulette Wheel [46].

These should be repeated until the predefined criteria are satisfied; the criterion in this paper has been taken as the completion of 100 iterations of the optimization process.

After introducing the proposed hybrid artificial intelligence paradigm and the components of it, a well-known evolutionary optimization technique, which is known as Genetic Algorithm, has been introduced in detail. In the next section, both of these algorithms have been implemented for optimizing the proposed RNP and the results have been analyzed and discussed in detail. It is important to know that since GA and other algorithms are not capable of adjusting the number of deployed antennas, in this paper GA has not been adopted as the sole optimization technique and it has been combined with RAE.

\section{Results and Discussion}

As discussed in the previous sections, the proposed dynamic working area in this paper is a conveyor belt with dimension of $3 \times 2 \mathrm{~m}$. 30 RFID passive dynamic tags mounted at the center of the parts, which have been moved along the conveyor with a constant speed equaling that of the belt, $0.3 \mathrm{~m} / \mathrm{s}$. For the first iteration of the optimization process, 30 RFID antennas, which is a number equal to the number of the tags, have been randomly deployed in the network.

The predefined number of deployed antennas has been reduced from 30 to 1 by the end of the proposed hybrid optimization process, by implementing both optimizing algorithms; however, this number has been calculated as 3 by GA (see Figure 19). After optimizing the number of antennas, in the next step, the coverage and interference of the RFID network, which both occur as a resultant of the deployed antennas, have been investigated.

The results shown in Figure 20 indicate that the RFID network coverage remains as $100 \%$ from the beginning until the end of both the optimization processes. 


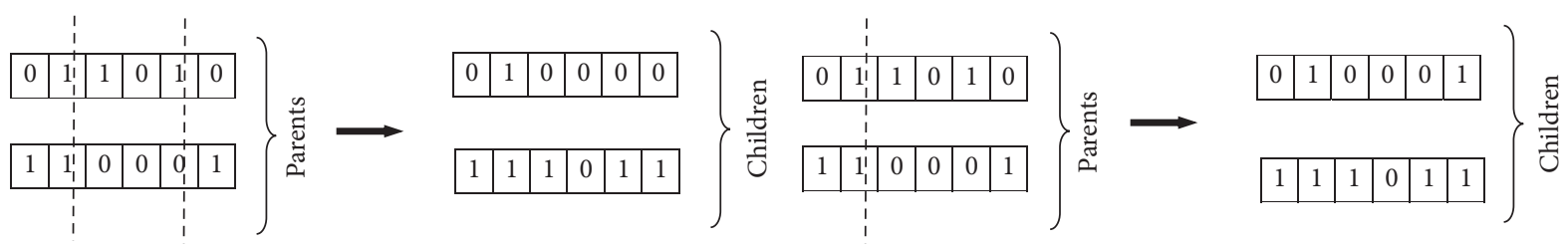

(a)

(b)

Figure 17: (a) Two points' Crossover and (b) single point crossover [46].

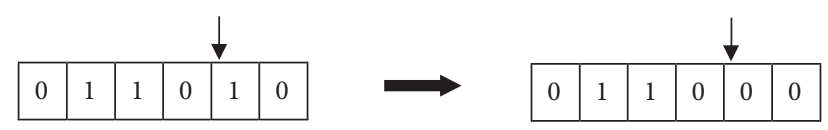

FIgURE 18: Example of mutation [46].

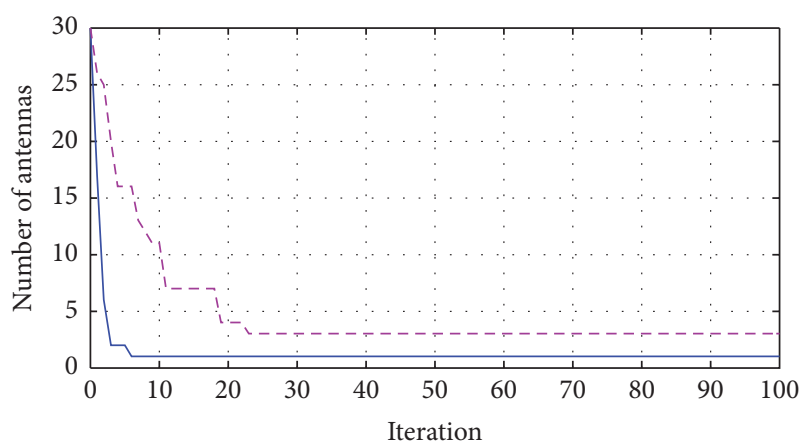

_ Hybrid algorithm; number of deployed antennas in the network in each iteration

- - - GA; number of deployed antennas in the network in each iteration

FIGURE 19: Calculated number of deployed antennas in the network by the proposed hybrid algorithm and GA in each iteration.

Since network coverage during both optimization processes remains as $100 \%$, then, contrary to the proposed static working area, investigating the network coverage results does not provide a clue to comparing the efficiency of the algorithms.

The interference of the RFID network occurs as a result of collision of the deployed antennas in the network. The ITF of the proposed RFID network from $-1000 \mathrm{dBm}$ has been reduced to $0 \mathrm{dBm}$ and $-183.66 \mathrm{dBm}$, by adopting GA and the proposed hybrid artificial intelligence paradigms, respectively (see Figure 21).

Therefore, the network interference results indicate that optimizing the RFID network by using the proposed hybrid algorithm, rather than the GA optimization technique, achieves more efficient numbers and positions of antennas.

Overall, the results indicate that utilizing the proposed hybrid artificial intelligence optimization technique rather than the GA optimization paradigm optimizes the RFID network better and provides a solution to RNP with the fewest antennas and lowest interference in the network.

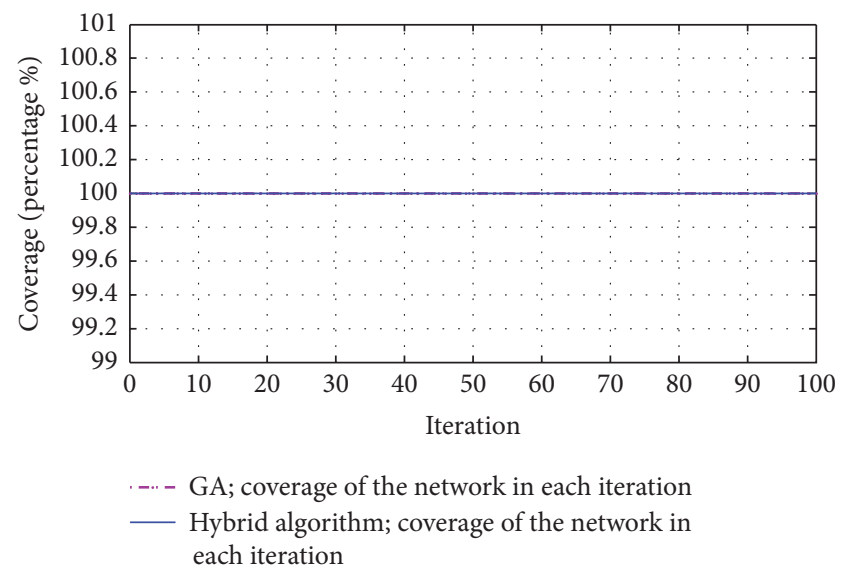

FIGURE 20: Calculated coverage of the rfid network by the proposed hybrid algorithm and GA in each iteration.

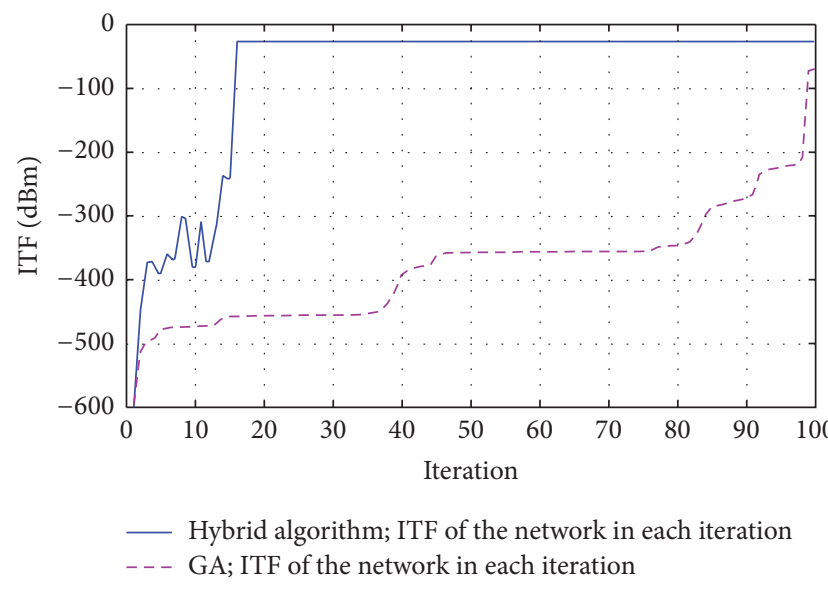

FIGURE 21: Calculated ITF of the network by the proposed hybrid algorithm and GA in each iteration.

By comparing the normalized fitness functions values of the two approaches, which have been shown in Figure 22, it is clear to see that the proposed hybrid algorithm has a superior performance than the GA.

Since the RFID network can reach full network coverage in fewer iterations, due to fewer deployment instances of antennas and less interference on the network, the proposed hybrid algorithm in comparison to GA is more cost effective 


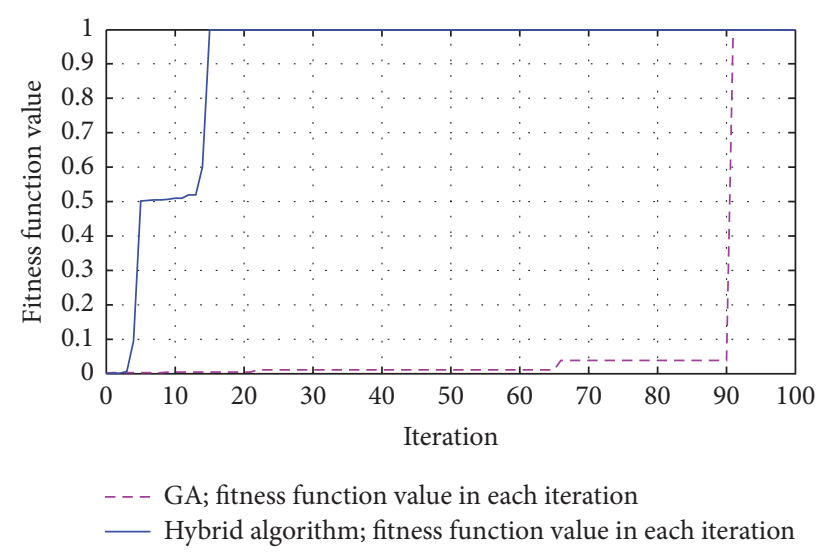

FIGURE 22: Normalized fitness functions values of the proposed hybrid algorithm and GA in each iteration.

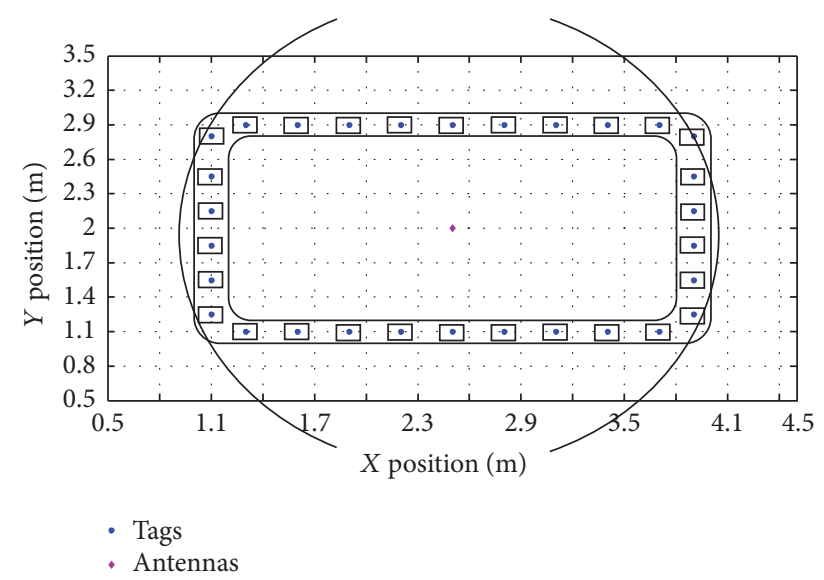

FIgURE 23: Optimized RFID network.

and a more time efficient technique. The optimized solution of RNP has been shown in Figure 23.

\section{Conclusion}

In this paper by combining two optimization paradigms, commonly known as RPLNN and RAE, a novel and efficient hybrid artificial optimization technique to deal with complex RFID Network Planning problems has been introduced and investigated. The proposed optimization technique is capable of adjusting any number of deployed RFID antennas in the network. The simulation based performance assessment has been performed for investigating the effectiveness of the hybrid algorithm over the GA. The simulation results show that the proposed hybrid algorithm has a superior performance as compared to the GA. It has been observed that the hybrid optimization technique for optimizing the RNP is more enhanced than GA, in the following aspects:

(i) The proposed hybrid algorithm has a faster convergence speed and it requires fewer iterations than GA.

(ii) The proposed hybrid algorithm has less computational complexity than GA. (iii) The results given by the proposed hybrid algorithm are more precise and cost effective.

In future studies, with an innovation in the proposed mathematical models of the RNP, it will be possible to model an RFID network that involves more criteria such as the quality of the network. A valuable future study would involve the utilization of more optimization techniques for static and dynamic networks to generate RFID networks.

\section{Conflicts of Interest}

The author declares that there are no conflicts of interest regarding the publication of this paper.

\section{References}

[1] A. V. Barenji, R. V. Barenji, and M. Hashemipour, "A framework for structural modelling of an RFID-enabled intelligent distributed manufacturing control system," South African Journal of Industrial Engineering, vol. 25, no. 2, pp. 48-66, 2014.

[2] A. C. P. Guédon, L. S. G. L. Wauben, D. F. de Korne, M. Overvelde, J. Dankelman, and J. J. van den Dobbelsteen, "A RFID Specific Participatory Design Approach to Support Design and Implementation of Real-Time Location Systems in the Operating Room," Journal of Medical Systems, vol. 39, no. 1, 2015.

[3] A. V. Barenji, An RFID-Based Distributed Control System for Flexible Manufacturing System, Eastern Mediterranean University (EMU)-Doğu Akdeniz Üniversitesi (DAÜ), 2013.

[4] Z. X. Guo, E. W. T. Ngai, C. Yang, and X. Liang, "An RFID-based intelligent decision support system architecture for production monitoring and scheduling in a distributed manufacturing environment," International Journal of Production Economics, vol. 159, pp. 16-28, 2015.

[5] A. V. Barenji, R. V. Barenji, and M. Hashemipour, "Flexible testing platform for employment of RFID-enabled multi-agent system on flexible assembly line," Advances in Engineering Software, vol. 91, pp. 1-11, 2016.

[6] R. V. Barenji, A. V. Barenji, and M. Hashemipour, "A multiagent RFID-enabled distributed control system for a flexible manufacturing shop," International Journal of Advanced Manufacturing Technology, vol. 71, no. 9-12, pp. 1773-1791, 2014.

[7] H. Chen, Y. Zhu, K. Hu, and T. Ku, "RFID network planning using a multi-swarm optimizer," Journal of Network and Computer Applications, vol. 34, no. 3, pp. 888-901, 2011.

[8] L. Ma, H. Chen, K. Hu, and Y. Zhu, "Hierarchical artificial bee colony algorithm for RFID network planning optimization," The Scientific World Journal, vol. 2014, Article ID 941532, 21 pages, 2014.

[9] L. Ma, K. Hu, Y. Zhu, and H. Chen, "Cooperative artificial bee colony algorithm for multi-objective RFID network planning," Journal of Network and Computer Applications, vol. 42, pp. 143$162,2014$.

[10] Q. Guan, Y. Liu, Y. Yang, and W. Yu, "Genetic approach for network planning in the RFID systems," in Proceedings of the ISDA 2006: Sixth International Conference on Intelligent Systems Design and Applications, pp. 567-572, chn, October 2006.

[11] Y. Yang, Y. Wu, M. Xia, and Z. Qin, "A RFID network planning method based on genetic algorithm," in Proceedings of the International Conference on Networks Security, Wireless 
Communications and Trusted Computing, NSWCTC 2009, pp. 534-537, chn, April 2009.

[12] M. Zhou and D. C. Ranasinghe, "A Novel Approach for Addressing Wandering Off Elderly Using Low Cost Passive RFID Tags," in Proceedings of the Mobile and Ubiquitous Systems: Computing, Networking, and Services: 10th International Conference, pp. 330-343, Tokyo, Japan, 2013.

[13] Y.-J. Gong, M. Shen, J. Zhang, O. Kaynak, W.-N. Chen, and Z.-H. Zhan, "Optimizing RFID network planning by using a particle swarm optimization algorithm with redundant reader elimination," IEEE Transactions on Industrial Informatics, vol. 8, no. 4, pp. 900-912, 2012.

[14] E. S. Robert and J. S. Patrick, "RFID: from concept to implementation," International Journal of Physical Distribution \& Amp, vol. 36, pp. 736-754, 2006.

[15] Z. Li and C. He, "Optimal scheduling-based RFID readerto-reader collision avoidance method using artificial immune system," Applied Soft Computing Journal, vol. 13, no. 5, pp. 25572568, 2013.

[16] R. J. Kuo, M. C. Shieh, J. W. Zhang, and K. Y. Chen, “The application of an artificial immune system-based back-propagation neural network with feature selection to an RFID positioning system," Robotics and Computer-Integrated Manufacturing, vol. 29, no. 6, pp. 431-438, 2013.

[17] T. Garcia-Valverde, A. Garcia-Sola, H. Hagras, J. A. Dooley, V. Callaghan, and J. A. Botia, "A fuzzy logic-based system for indoor localization using WiFi in ambient intelligent environments," IEEE Transactions on Fuzzy Systems, vol. 21, no. 4, pp. 702-718, 2013.

[18] O. Botero and H. Chaouchi, "RFID network topology design based on Genetic Algorithms," in Proceedings of the 2011 2nd IEEE RFID Technologies and Applications Conference, RFIDTA 2011, Collocated with the 2011 IEEE MTT-S International Microwave Workshop Series on Millimeter Wave Integration Technologies, IMWS 2011, pp. 300-305, esp, September 2011.

[19] H. Chen, Y. Zhu, L. Ma, and B. Niu, "Multiobjective RFID network optimization using multiobjective evolutionary and swarm intelligence approaches," Mathematical Problems in Engineering, vol. 2014, Article ID 961412, 13 pages, 2014.

[20] I. Bhattacharya and U. K. Roy, "Optimal placement of readers in an RFID network using particle swarm optimization," International Journal of Computer Networks \& Amp, vol. 2, pp. 225-234, 2010.

[21] F. Han and Q. Jie, "Optimal RFID networks planning using a hybrid evolutionary algorithm and swarm intelligence with multi-community population structure," in Inproceeding of the 2012 14th International Conference on Advanced Communication Technology (ICACT), pp. 1063-1068, 2012.

[22] A. Nawawi, K. Hasnan, and S. Ahmad Bareduan, "Correlation between RFID network planning (RNP) parameters and particle swarm optimization (PSO) solutions," Applied Mechanics and Materials, vol. 465-466, pp. 1245-1249, 2014.

[23] S. Lu and S. Yu, "A fuzzy $k$-coverage approach for RFID network planning using plant growth simulation algorithm," Journal of Network and Computer Applications, vol. 39, pp. 280-291, 2014.

[24] http://www.cisco.com.

[25] C.-H. Wang and S.-W. Tsai, "Optimizing bi-objective imperfect preventive maintenance model for series-parallel system using established hybrid genetic algorithm," Journal of Intelligent Manufacturing, vol. 25, no. 3, pp. 603-616, 2014.

[26] B. L. MacCarthy, C. Blome, J. Olhager et al., "Supply chain evolution-theory, concepts and science," International Journal of Operations \& Amp; Production Management, vol. 36, pp. 1696-1718.

[27] D. Marshall, R. Metters, and M. Pagell, "Changing a Leopard's Spots: A New Research Direction for Organizational Culture in the Operations Management Field," Production and Operations Management, vol. 25, no. 9, pp. 1506-1512, 2016.

[28] K. Jung, K. Morris, K. W. Lyons, S. Leong, and H. Cho, "Performance challenges identification method for smart manufacturing systems," National Institute of Standards and Technology, NISTIR, vol. 8108, 2016.

[29] L. Atzori, A. Iera, and G. Morabito, "The internet of things: a survey," Computer Networks, vol. 54, no. 15, pp. 2787-2805, 2010.

[30] S. Bhatia, A. Chauhan, and V. K. Nigam, The Internet of Things: A Survey on Technology and Trends, The Internet of Things, A Survey on Technology and Trends, 2016.

[31] D. M. Segura Velandia, N. Kaur, W. G. Whittow, P. P. Conway, and A. A. West, "Towards industrial internet of things: Crankshaft monitoring, traceability and tracking using RFID," Robotics and Computer-Integrated Manufacturing, vol. 41, pp. 66-77, 2016.

[32] S. De Mel, D. Herath, D. McKenzie, and Y. Pathak, "Radio frequency (un)identification: Results from a proof-of-concept trial of the use of RFID technology to measure microenterprise turnover in Sri Lanka," Development Engineering, vol. 1, pp. 4-11, 2016.

[33] S. J. Simske, J. S. Aronoff, M. P. Gore, R. A. Stamey, and M. Eagan, in RFID antenna and $2 D$ barcode, Google Patents edition, 2016.

[34] S. Gupta, C. Koulamas, and G. J. Kyparisis, "E-business: A review of research published in production and operations management (1992-2008)," Production and Operations Management, vol. 18, no. 6, pp. 604-620, 2009.

[35] Z. Irani, A. Gunasekaran, and Y. K. Dwivedi, "Radio frequency identification (RFID): Research trends and framework," International Journal of Production Research, vol. 48, no. 9, pp. 24852511, 2010.

[36] G. Q. Huang, Y. F. Zhang, X. Chen, and S. T. Newman, "RFIDenabled real-time wireless manufacturing for adaptive assembly planning and control," Journal of Intelligent Manufacturing, vol. 19, no. 6, pp. 701-713, 2008.

[37] C. M. Roberts, "adio frequency identification (RFID)," Computers \& Amp, vol. 25, pp. 18-26, 2006.

[38] B. Khoo, "RFID As an enabler of the internet of things: Issues of security and privacy," in Proceedings of the 2011 IEEE International Conference on Internet of Things, iThings 2011 and 4th IEEE International Conference on Cyber, Physical and Social Computing, CPSCom 2011, pp. 709-712, chn, October 2011.

[39] C. A. Balanis, Antenna theory: analysis and design, John Wiley \& Amp, 2016.

[40] U. Azad, H. C. Jing, and Y. E. Wang, "Link budget and capacity performance of inductively coupled resonant loops," IEEE Transactions on Antennas and Propagation, vol. 60, no. 5, pp. 2453-2461, 2012.

[41] J. R. Smith, Wirelessly Powered Sensor Networks and Computational RFID, Springer New York, New York, NY, 2013.

[42] J. A. Shaw, "Radiometry the Friis transmission equation," American Journal of Physics, vol. 81, no. 1, pp. 33-37, 2012.

[43] T. B. Ludermir and W. R. de Oliveira, "Weightless neural models," Computer standards \& Amp; interfaces, vol. 16, pp. 253-263, 1994. 
[44] A. Azizi, A. Vatankhah Barenji, and M. Hashmipour, "Optimizing radio frequency identification network planning through ring probabilistic logic neurons," Advances in Mechanical Engineering, vol. 8, 2016, 1687814016663476.

[45] A. Azizi, A. Barenji, R. Barenji, and M. Hashemipour, "Modeling Mechanical Properties of FSW Thick Pure Copper Plates and Optimizing It Utilizing Artificial Intelligence Techniques," Sensor Netw Data Commun, vol. 5, p. 2, 2016.

[46] A. Ashkzari and A. Azizi, "Introducing genetic algorithm as an intelligent optimization technique," Applied Mechanics and Materials, vol. 568-570, pp. 793-797, 2014.

[47] M. Tao, S. Huang, Y. Li, M. Yan, and Y. Zhou, "SA-PSO based optimizing reader deployment in large-scale RFID Systems," Journal of Network and Computer Applications, vol. 52, pp. 90100, 2015.

[48] W.-k. Kan and I. Aleksander, "A probabilistic logic neuron network for associative learning," in Neural computing architectures, A. Igor, Ed., pp. 156-171, MIT Press, 1989.

[49] L. Zeng, B. Benatallah, M. Dumas, J. Kalagnanam, and Q. Z. Sheng, "Quality driven web services composition," in Proceedings of the 12th international conference on World Wide Web, pp. 411-421, 2003.

[50] M. B. Menhaj and N. Seifipour, "Function optimization by RPLNN," in Neural Networks, 2002, pp. 1522-1527.

[51] J. Austin, "A review of RAM based neural networks," in Proceedings of the Fourth International Conference, pp. 58-66, 1994.

[52] I. Aleksander, M. De Gregorio, F. M. G. França, P. M. V. Lima, and H. Morton, "A brief introduction to Weightless Neural Systems," in ESANN, pp. 299-305, 2009.

[53] M. R. Berthold and J. Diamond, "Constructive training of probabilistic neural networks," Neurocomputing, vol. 19, no. 1-3, pp. 167-183, 1998.

[54] J. Dias, H. Rocha, B. g. Ferreira, and M. d. Lopes, "A genetic algorithm with neural network fitness function evaluation for IMRT beam angle optimization," Central European Journal of Operations Research (CEJOR), vol. 22, no. 3, pp. 431-455, 2014.

[55] R. Martínez-Soto, O. Castillo, and J. R. Castro, "Genetic Algorithm Optimization for Type-2 Non-singleton Fuzzy Logic Controllers," in Recent Advances on Hybrid Approaches for Designing Intelligent Systems, O. Castillo, P. Melin, W. Pedrycz, and J. Kacprzyk, Eds., pp. 3-18, Cham: Springer International Publishing edition.

[56] K.-F. Man, K. S. Tang, and S. Kwong, Genetic Algorithms: Concepts and dEsigns, Springer Science \& Amp; Business Media, 2012.

[57] E. Semenkin and M. Semenkina, "Self-configuring Genetic Algorithm with Modified Uniform Crossover Operator," in Inproceeding of the Advances in Swarm Intelligence: Third International Conference ICSI 2012, Proceedings., I. Part, Y. Tan et al., Eds., pp. 414-421, Shenzhen, China, 2012. 


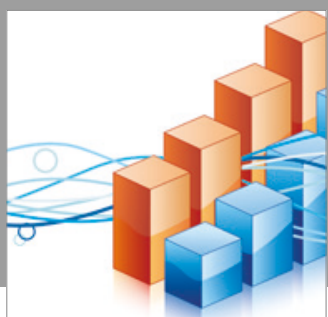

Advances in

Operations Research

vatersals

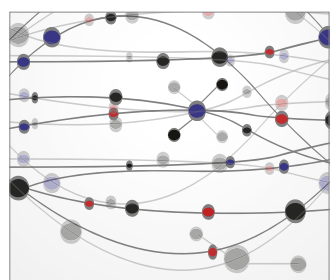

\section{The Scientific} World Journal
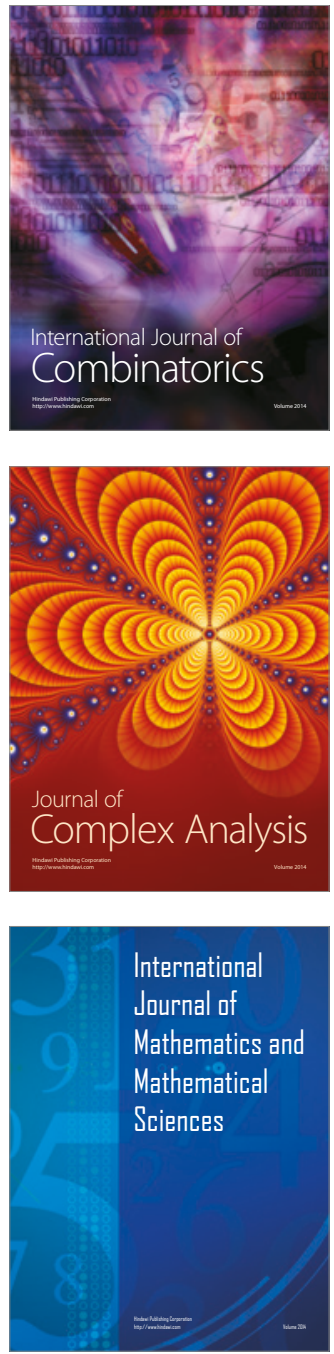
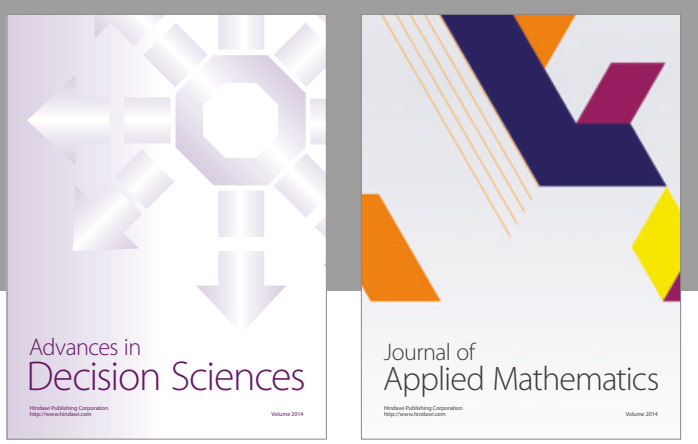

Algebra

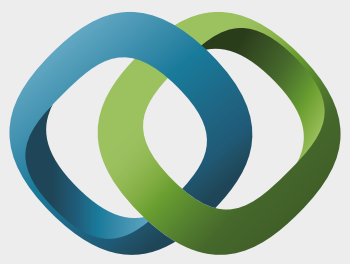

\section{Hindawi}

Submit your manuscripts at

https://www.hindawi.com
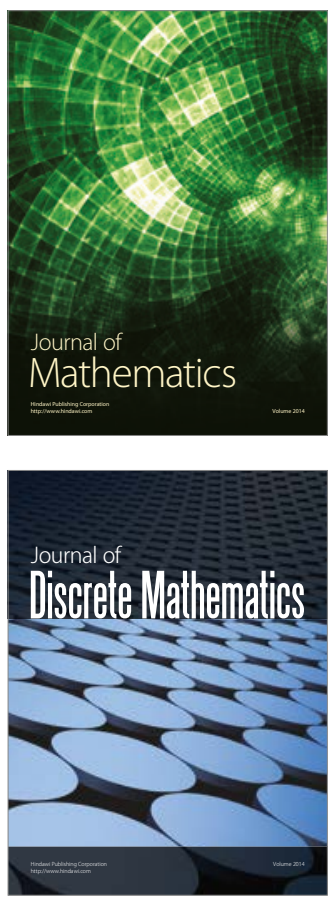

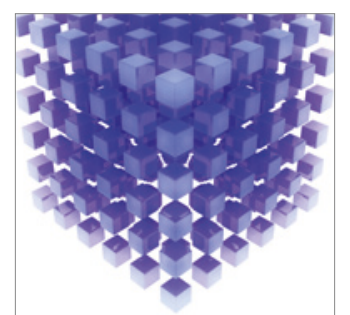

Mathematical Problems in Engineering
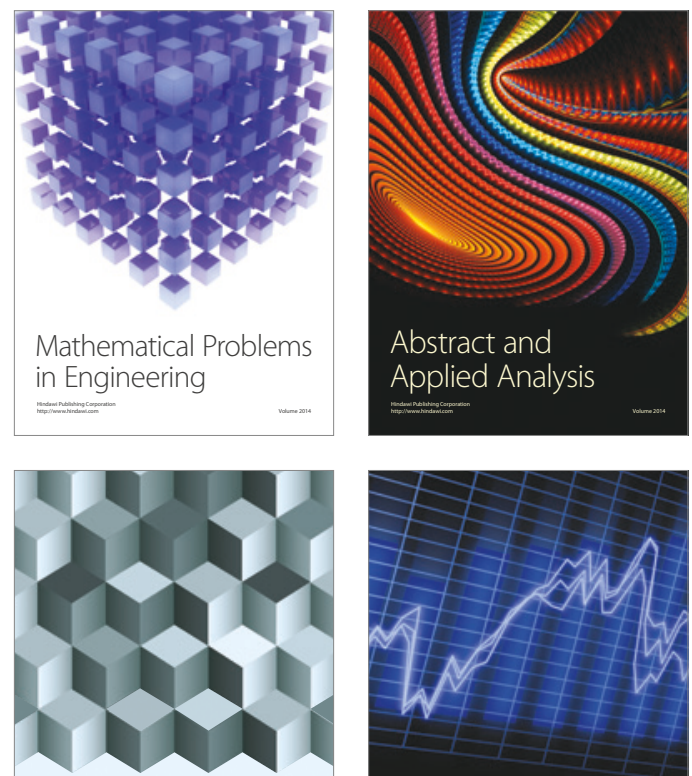

Journal of

Function Spaces

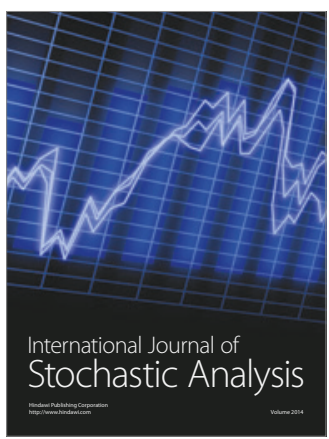

Probability and Statistics
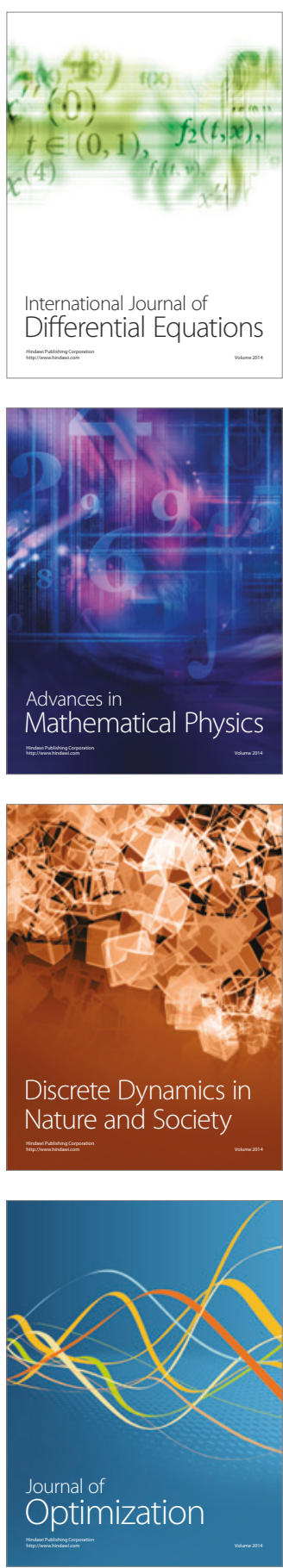